\title{
STATISTICAL CONSIDERATION ON SAMPLING ERRORS IN TOTAL BACTERIAL CELL COUNT*
}

\author{
KOITI TAKAHASI \\ Institute of Statistical Mathematics, Tokyo \\ SETSUJI ISHIDA AND MASAMI KUROKAWA \\ Department of General Biologics Control, National Institute \\ of Health, Tokyo
}

(Received: April 28th, 1964)

Procedures proposed for the determination of the total number of cells in a given volume of a bacterial suspension are usually classified into three categories as follows (Olitzki, 1960 ; Joó, 1960);

1) the method of direct counting under the microscope of a stained preparation of a known volume on a slide.

2) the method of direct microscopic counting in a counting chamber, which involves counting the organisms in a certain number of microscopic fields selected at random and calculating the total fluid volume in terms of the number of microscopic fields observed. This type of method is referred to shortly as "chamber method" in what follows, and

3) the method represented by Wright's method, in which a known quantity of a bacterial cell suspension to be tested is added to a standardized particle suspension and the cell concentration of a test suspension is estimated from the ratio of number of the bacterial cells to the particles of the standard in a constant number of microscopic fields. This sort of method is referred to as " ratio method" in this paper.

Since the first method in the above has an essential weakness in controlling a minute volume of the suspension, it was not included within the scope of the present paper.

Though the chamber method is generally considered as the most precise one, it is not always practical in cases where many countings are to be carried out simultaneously or periodically with relatively short intervals, because a number of counting chambers are indispensable. On the contrary, the ratio method is practically, as well as economically, convenient, since the slides can be preserved, stained and examined whenever a retesting occasion arises. It is, in general, considered that the weakness of this method lies in its difficulty to obtain a sufficiently homogeneous distribution of two kinds of particles on a slide, though this weakness will be prevented by using as the standard a suspension of particles of the same physical properties as the particles to be tested. On the other hand, when the smearing is made on a slide, another kind of heterogeneity of distribution may arise, because of the difficulty of smearing the mixed suspension uniformly on

\footnotetext{
* Supported by a grant from the Ministry of Education. 高橋宏一(統計数理研究所)

石田説而・黑川正身（国立予防徫生研究所一般検定部）
} 
a slide. In such a case the mean value of the numbers of cells per unit square* will be significantly variable with fields**.

Considerations on sampling errors in the total cell count by the chamber method usually have been based on the Poisson distribution, except the report of Jones et al. (1948), while there has been no report on the ratio method. The authors, however, have experienced that the Poisson distribution had not always been fitted to the above cases. Jones et al. reported that with a cell suspension containing some clumps of cells frequencies of small numbers of cells per microscopical unit square followed a negative binomial distribution. Moreover, with unhomogeneous smearing the distribution of numbers of cells per unit square will deviate from the Poisson distribution, too.

Therefore, in considering the distribution of numbers of cells per unit square, it is necessary to include not only the cases where clumps are contained but also such cases where the distribution is not homogeneous. Besides, the considerations should be extended to the ratio method, where there is a joint distribution of the numbers of the test cells and that of the reference cells per unit square.

The above mentioned is the basic points of our investigations in the present report. In this paper, theoretical interpretations on the nature of the distributions and analysis of experimental data are presented (Part I). Considerations on the sample size in the ratio method are also revealed (Part II).

\section{Part I. Nature of the Distributions Theoretical Considerations}

The following considerations on the distribution of numbers of cells per unit square were divided into four cases for simplification; A) no clump*** exists and the mean of the numbers of cells per unit square is not variable with fields, B) no clump exists and the mean is variable with fields, C) clumps exist and the mean is not variable, and D) clumps exist and the mean is variable. Furthermore, each case was divided into two parts, i) and ii), corresponding to the chamber method and the ratio method, respectively.

(A) No clump exists and the mean is not variable.

i) The chamber method: Let $X$ be the random variable denoting the number of cells per unit square. The variable $X$ will follow the Poisson distribution with mean $\lambda$,

$$
\operatorname{Pr}\{X=k\}=\frac{e^{-\lambda} \lambda^{k}}{k !},(k=0,1,2, \cdots \cdots ; \lambda>0)
$$

ii) The ratio method: Let $S$ and $T$ be the random variables denoting the number of the reference cells and that of the test cells per unit square, respectively. The random vectors $(S, T)$ will follow the direct product of two independent Poisson distributions,

$$
\operatorname{Pr}\{S=k, T=l\}=\frac{e^{-\lambda_{1}} \lambda_{1}^{k}}{k !} \frac{e^{-\lambda_{2}} \lambda_{2}^{l}}{l !}\left(k, l=0,1,2, \cdots \cdots ; \lambda_{1}, \lambda_{2}>0\right) .
$$

(B) No clump exists and the mean is variable.

\footnotetext{
*, ** The terms "unit square" and "field" in the above text and on the following pages are used to convey two entirely different meanings as follows; "Unit square" denotes the smallest ruled square on the chamber or the glass disk inserted in the eyepiece.

"Field" is used to denote a small field on the slide corresponding to a group of unit squares.

*** For the convenience of description, the term "clump" was used in the text to denote cell aggregates composed of two or more cells, while in the mathematical formulae each isolated cell was reckoned as a "clump" composed of a single cell.
} 
In this case, the parameter $\lambda$ denoting the mean in the Poisson distribution is a random variable. Let us assume that the random variable exhibits a Gamma distribution, whose probability density function $f(\lambda)$ is given by

$$
f(\lambda)=\frac{1}{\gamma \Gamma(p)} e^{-\frac{\lambda}{\gamma}}\left(\frac{\lambda}{\gamma}\right)^{p-1},(\lambda>0, p>0, \gamma>0) .
$$

This assumption may be applicable because the Gamma distribution having two parameters is flexible.

i) If the number of cells per unit square follows a Poisson distribution with the parameter $\lambda$ which is a random variable with the probability density function (3), then the resulting compound distribution,

$$
\operatorname{Pr}\{X=k\}=\frac{\Gamma(p+k)}{\Gamma(p) k !}\left(\frac{1}{1+\gamma}\right)^{p}\left(\frac{\gamma}{1+\gamma}\right)^{k},(k=0,1,2, \cdots \cdots),
$$

is a negative binomial distribution (or Greenwood-Yule distribution) (Gurland, 1959).

ii) We can a'so apply the above consideration to the ratio method. If the assumptions, $\lambda_{1}=\rho_{1} \lambda, \lambda_{2}=\rho_{2} \lambda$, where $\rho_{1}, \rho_{2}$ are positive constants and $\lambda$ is a random variable with the probability density function (3), are given to the parameters in (2), then the resulting compound two-dimentional distribution,

$$
\operatorname{Pr}\{S=k, T=l\}=\frac{1}{\gamma^{p} \Gamma(p)} \frac{\Gamma(\gamma+k+1)}{k ! l !} \frac{\rho_{1}^{k} \rho_{2}^{l}}{\begin{array}{r}
\left(\frac{1}{r}+\rho_{1}+\rho_{2}\right)^{p+k+1} \\
(k, l=0,1,2, \cdots \cdots),
\end{array}}
$$

is a so-called bivariate negative binomial distribution (Arbous and Sichell, 1954).

(C) Clumps exist and the mean is not variable.

i) Let us assume that the number of clumps per unit square follows a Poisson distribution and the number of cells per clump a discrete distribution. Moreover, if the latter is assumed to be a logarithmic series distribution (Jones et al., 1948), whose probability law is given by

$$
\operatorname{Pr}\{Y=h\}=\frac{-1}{\log (1-q)} \cdot \frac{q^{h}}{h},(h=1,2,3, \cdots \cdots, 0<q<1),
$$

where $Y$ is the random variable denoting the number of cells per clump, then the distribution of numbers of cells per unit square will be a negative binomial,

$$
\operatorname{Pr}\{X=k\}=\frac{\Gamma(m+k)}{\Gamma(m) k !} \alpha^{m} \cdot(1-\alpha)^{k},(k=0,1,2, \cdots \cdots),
$$

where

$$
m=\frac{-\lambda}{\log (1-q)} ; \alpha=1-q \quad \text { (Quenouille, 1949). }
$$

ii) Let $q_{1}$ and $q_{2}$ be the parameter $q$ in (6) for the reference cells and the test cells, respectively, and $\lambda_{1}$ and $\lambda_{2}$ the parameters $\lambda$ for the reference cells and the test cells, respectively, then the distribution of $(S, T)$ will be given by

$$
\begin{array}{r}
\operatorname{Pr}\{S=k, T=l\}=\frac{\Gamma\left(m_{1}+k\right)}{\Gamma\left(m_{1}\right) k !} \alpha_{1}^{m_{1}}\left(1-\alpha_{1}\right)^{k} \cdot \frac{\Gamma\left(m_{2}+l\right)}{\Gamma\left(m_{2}\right) l !} \alpha_{2}^{m_{2}}\left(1-\alpha_{2}\right)^{l}, \\
\left(k, l=1,2,3, \cdots \cdots ; m_{i}=\frac{-\lambda_{i}}{\log \left(1-q_{i}\right)}, \alpha_{i}=1-q_{i}, i=1,2\right) .
\end{array}
$$

(D) Clumps exist and the mean is variable.

In this case, the probability law of the distribution of numbers of cells can be obtained 
by combining the considerations in (B) and (C). This problem is not referred to in this paper, because it is too much complicated. And the mathematical models in the above will provide practically satisfactory informations.

\section{MATERIALS AND METHODS}

Bacteria: A strain of Escherichia coli, B, was used, which was obtained by the courtesy of Dr. R. Nakaya, Department of Bacteriology I, National Institute of Health, Tokyo.

Cell counting chamber: Petroff-Hausser bacteria counter (A. H. Thomas Co., Philadelphia) (the unit square is $1 / 400$ sqmm and $1 / 50 \mathrm{~mm}$ deep and there are 400 unit squares) was used.

Chamber method: The ordinary method for counting bacterial cells with a chamber was applied. Cells were counted either after staining with Gentian violet solution or without staining.

Ratio method: It is reasonably assumed that erythrocytes and bacterial cells will be affected differently by the surface-tension forces during the drying of the suspension, so that the distributions of the two populations of particles would be different from each other. Thornton and Gray (1934), in estimation of the number of bacteria in unit amount of soil, proposed a revised method using a standardized suspension of indigo particles which were similar to bacteria in the size and density.

A more homogeneous distribution of both kinds of particles could be obtained, if the same species of cells is used. A standardized stained cell suspension of the same strain as the cells to be tested was used as the reference in the present study. The reference cell suspension was prepared as follows. A logarithmic phase culture of the strain on a shaking machine was harvested, stained with Gentian violet solution for several hours at room temperature, washed three times with saline and then suspended in saline to a desired concentration. Possible clumps of cells were disrupted as thoroughly as possible by a mild sonic oscillation. The concentration was standardized by the chamber method.

The ratio method was carried out as follows. The cell suspension to be counted was mixed with the reference suspension so that the ratio of concentration of the two cell suspensions was nearly unity. The mixture was smeared homogeneously on a slide as far as possible, dried, fixed and stained with Pfeiffer's solution. The cells on the slide were stained in two different colors, the reference in purple and the test in light red. The numbers of the test cells and those of the reference cells were simultaneously counted for each unit square of an ocular micrometer inserted in the eyepiece. The cell concentration of the test suspension was computed from the ratio of the total number of the test cells to the reference cells.

If there are clumps composed of two cells or more, the number of clumps of each color per unit square and the number of cells per clump were recorded. The squares counted were selected randomly throughout the smear.

Statistical procedures: Parameters of a distribution in attempting to fit observed data were estimated by the maximum likelihood method (Bliss and Fisher, 1953). For testing the goodness of fit to the observed frequencies by a theoretical distribution the $\chi^{2}$ statistic was applied.

Table 1. Fitting the Poisson distributions to data from the chamber method

\begin{tabular}{rcccc}
\hline $\begin{array}{c}\text { Exp. } \\
\text { No. }\end{array}$ & $\begin{array}{c}\text { Number of unit } \\
\text { squares counted }\end{array}$ & $\begin{array}{c}\text { Mean of cells } \\
\text { per unit square }\end{array}$ & Variance & Variance/Mean \\
\hline 1 & 160 & 4.11 & 5.17 & $1.26^{*}$ \\
2 & 160 & 3.76 & 3.06 & 0.81 \\
3 & 400 & 4.29 & 10.17 & $2.37^{* *}$ \\
4 & 400 & 5.37 & 11.77 & $2.19^{* *}$ \\
5 & 80 & 3.74 & 3.84 & 1.03 \\
6 & 160 & 4.64 & 7.74 & $1.67^{* *}$ \\
7 & 400 & 4.84 & 6.25 & $1.29^{* *}$ \\
\hline
\end{tabular}

* Significant at the $5 \%$ level of probability.

** Significant at the $1 \%$ level of probability. 


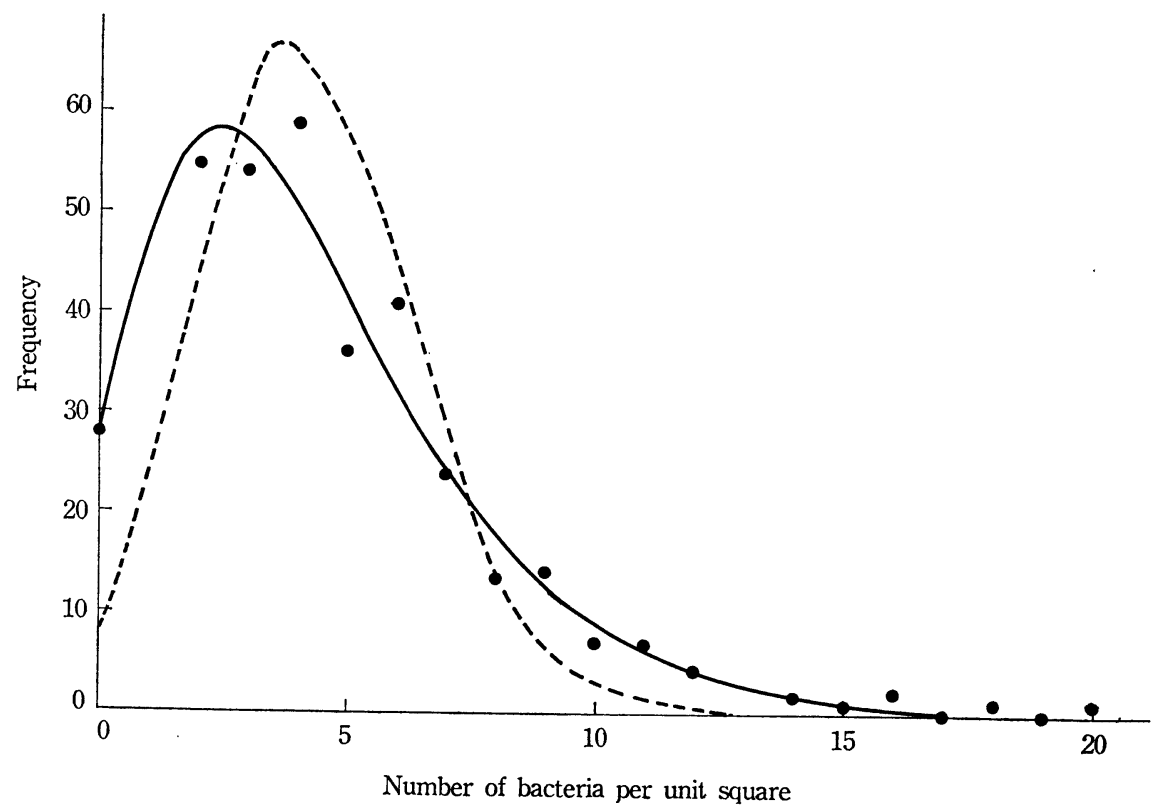

Fig. 1. Fitting the negative binomial distribution and the Poisson distribution to an observed distribution from the chamber method.

- Observed value

Theoretical curve based on

- Negative binomial distribution

-.. Poisson distribution

Total $=359, \quad$ Mean $=4.293$

Estimates $\hat{\gamma}=\hat{\lambda}=4.298, \hat{\mathbf{p}}=3.139$ (c. f. equation (4))

Poisson : $\chi^{2}=342 \quad\left(\chi_{9}{ }^{2}(0.05)=16.919\right)$,

Negative binomial : $\chi^{2}=9.76 \quad\left(\chi_{11}^{2}(0.05)=19.675\right)$

\section{EXPERIMENTAL RESULTS}

\section{The chamber method.}

An example of fitting the Poisson and the negative binomial distributions to an observed distribution of numbers of cells per unit square obtained by the chamber method is shown in Fig. 1. In this example, the negative binomial was obviously more close to the observed frequencies than the Poisson.

Several experimental results from the chamber method including the data shown in Fig. 1 are summarized in Table 1. Some of those (for example Expts. No. 3 and 4) clearly deviated from the Poisson distribution.

2. The ratio method.

As no suspension without any clump has been available, the test for goodness of fit of the bivariate negative binomial distribution, the formula (5), could not be made to any actual data from the ratio method.

The data from the ratio method in Table 2, though there were a few clumps, was assumed as a sample from a bivariate negative binomial distribution and the parameters were estimated. As seen in the table, theoretical distribution thus obtained was fairly 
close to the observed joint frequency, though the fit was not so good, probably because the existence of clumps was neglected in the analysis.

It is known that if a random vector $(X, Y)$ follows a bivariate negative binomial distribution each of its two marginal distributions, $X$ and $Y$, and the distribution of sum of two variables $X+Y$ follow the negative binomial distribution, respectively. Thus the theoretical distributions of the sum of two variables and each of the two variables were demonstrated to be satisfactorily fitted to the data, respectively (Fig. 2).

Table 2. An example of the ratio method. Frequency distribution of numbers of reference cells and test cells per unit square

\begin{tabular}{|c|c|c|c|c|c|c|c|c|}
\hline & & \multicolumn{6}{|c|}{ Number of the reference cells per unit square } & \multirow{2}{*}{ Total } \\
\hline & & $0-4$ & $5-9$ & $10-14$ & $15-19$ & $20-24$ & $30-34$ & \\
\hline \multirow{6}{*}{$\begin{array}{l}\text { Number of the } \\
\text { test cells per } \\
\text { unit square }\end{array}$} & $3-4$ & $\begin{array}{c}138 \\
(146.2)\end{array}$ & $\begin{array}{c}114 \\
(113.5)\end{array}$ & $\begin{array}{c}15 \\
(10.9)\end{array}$ & $1^{*}$ & & & 268 \\
\hline & $5-9$ & $\begin{array}{c}172 \\
(171.5)\end{array}$ & $\begin{array}{c}268 \\
(287.5)\end{array}$ & $\begin{array}{c}66 \\
(60.6)\end{array}$ & $4^{*}$ & & & 528 \\
\hline & $10-14$ & $\begin{array}{c}39 \\
(27.9)\end{array}$ & $\begin{array}{c}98 \\
(100.0)\end{array}$ & $\begin{array}{c}30 \\
(44.2)\end{array}$ & $\begin{array}{c}7 \\
(6.8)\end{array}$ & $13(9.2)^{* *}$ & & 174 \\
\hline & $15-19$ & $1^{*}$ & $\begin{array}{c}8 \\
(11.7)\end{array}$ & $\begin{array}{c}14 \\
(10.0)\end{array}$ & $2^{*}$ & & & 25 \\
\hline & $20-24$ & & $1^{*}$ & $3^{*}$ & & & & 4 \\
\hline & $25-29$ & & & & & & $1 *$ & 1 \\
\hline Total & & 350 & 507 & 128 & 14 & & 1 & 1000 \\
\hline
\end{tabular}

Figures in parenthesis are the expected frequencies based on the bivariate negative binominal distribution.

*, ** Figures with an asterisk were combined to make the figure with two asterisks.

$\chi^{2}=15.858 \quad\left(\chi_{9}{ }^{2}(0.05)=16.92\right)$

3. The distribution of numbers of cells per clump.

An example of fitting the logarithmic series distribution to an observed frequency of numbers of cells per clump is given in Table 3 and Fig. 3. The fit was sufficiently satisfactory

\section{The distribution of numbers of clumps per unit square.}

An example of fitting the normal distribution to an observed frequency of number of clumps per unit square (Table 4), of which the mean was so large that a normal distribution should give a good approximation to the Poisson, is shown in Fig. 4. As seen in the figure, there was a fairly good fit of the theoretical line on a normal probability paper to the data.

\section{Part II. Calculation of Sample Size in the Ratio Method}

The more clumps the suspensions contain, the larger the sample size will be required in order to estimate the above ratio with a given precision. We shall now turn our attention to the sample size. In Part II. equations are presented by which approximate values of the sample size in the ratio method are given. The formula was led under an assumption that there was no clump containing both the reference and the test cells simultaneously. Note that each isolated cell was reckoned as a "clump" composed of 


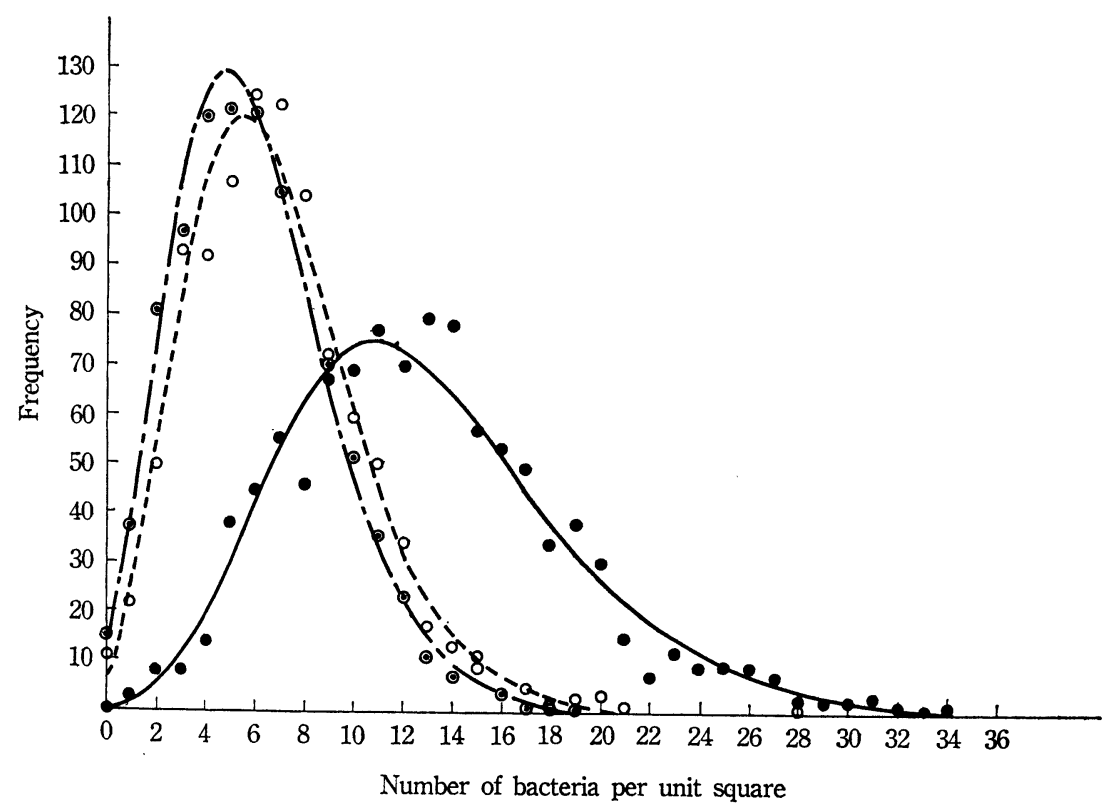

Fig. 2. Fitting the negative binomial distributions to an observed distribution from the ratio method.

Theoretical curve based on the negative binomial distribution

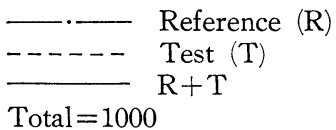

Total $=1000$

Reference $\chi^{2}=9.04 \quad\left(\chi_{15}^{2}(0.05)=24.996\right)$

Test $\quad \chi^{2}=16.08 \quad\left(\chi_{16}^{2}(0.05)=26.295\right)$

$\mathrm{R}+\mathrm{T} \quad \chi^{2}=30.05 \quad\left(\chi_{25}{ }^{2}(0.05)=37.652\right)$

Table 3. An example of fitting the logarithmic distribution to an observed frequency of numbers of cells per clump

\begin{tabular}{ccc}
\hline $\begin{array}{c}\text { Number of bacteria } \\
\text { per clump }\end{array}$ & Frequency & Theoretical frequency \\
\hline 1 & 1507 & 1507 \\
2 & 393 & 398 \\
3 & 138 & 140 \\
4 & 62 & 56 \\
5 & 27 & 23 \\
6 & 8 & 10 \\
7 & 8 & 5 \\
8 & 0 & 1 \\
9 & 0 & 1 \\
10 & 0 & 0 \\
11 & 0 & 2143 \\
\hline
\end{tabular}

Mean $=1.490 \quad \hat{\mathrm{q}}=0.528$

$\chi^{2}=7.6\left(\chi_{6}{ }^{2}(0.05)=12.59\right)$ 


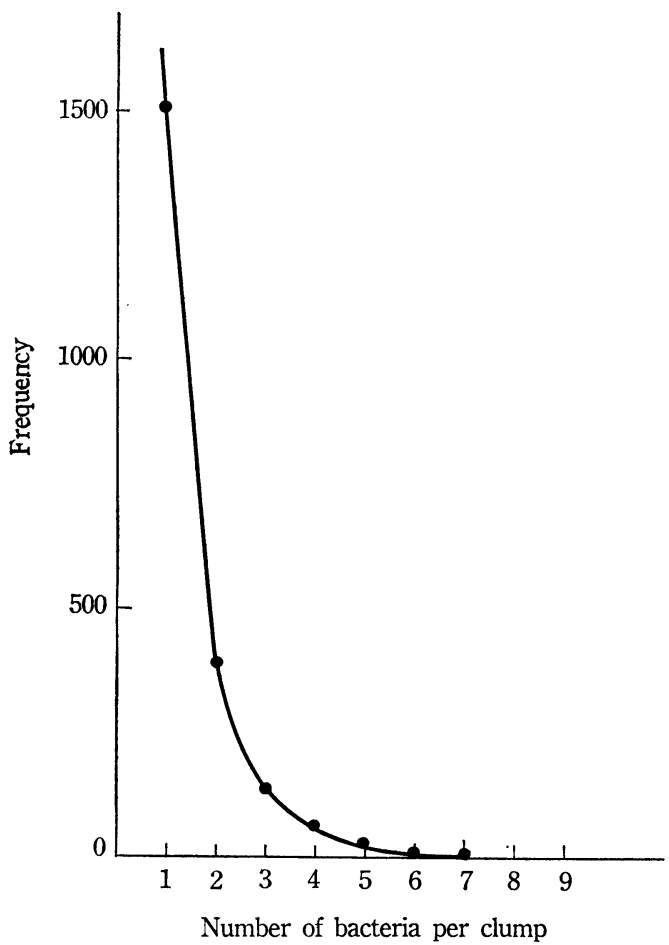

Fig. 3. Fitting the logarithmic distribution to an observed frequency of numbers of bacteria per clump.

- Observed value

Theoretical curve based on the logarithmic distribution $\chi^{2}=7.6 \quad\left(\chi_{6}{ }^{2}(0.05)=12.592\right)$

Table 4. An example of the frequency distribution of numbers of clumps per unit square

\begin{tabular}{ccc}
\hline $\begin{array}{c}\text { Number of clumps } \\
\text { per square }\end{array}$ & Frequency & Expected frequency \\
\hline-16 & 1 & 1 \\
$17-19$ & 4 & 2 \\
$20-22$ & 7 & 6 \\
$23-25$ & 17 & 11 \\
$26-28$ & 20 & 22 \\
$29-31$ & 25 & 19 \\
$32-34$ & 12 & 11 \\
$35-37$ & 7 & 6 \\
$38-40$ & 3 & 2 \\
$41-43$ & 1 & 1 \\
$44-46$ & 3 & 0 \\
$47-$ & 0 & \\
\hline
\end{tabular}

$\chi^{2}=9.24\left(\chi_{6}^{2}(0.05)=12.59\right)$ 


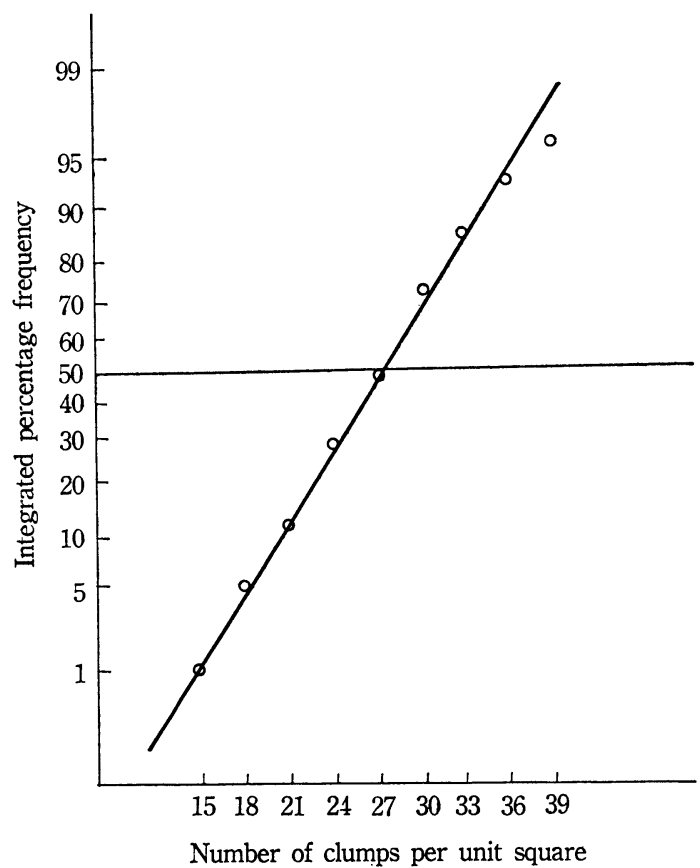

Fig. 4. Fitting the normal distribution to an observed frequency of numbers of clumps per unit square.

Observed value

Theoretical line based on the normal distribution $\chi^{2}=9.24 \quad\left(\chi_{6}{ }^{2}(0.05)=12.59\right)$

a single cell.

Let $\mathrm{s}$ and $\mathrm{t}$ be the means of numbers of cells per clump of the reference and the test cells, respectively, and $\sigma_{s}{ }^{2}$ and $\sigma_{t}{ }^{2}$ the variances for the reference and the test cells, respectively. And let $X$ be the random variable representing the number of the reference cells when $N$ clumps are counted, and $Y$ that of the test cells. Then the expectations of $X$ and $Y, \mathrm{E}(X)$ and $\mathrm{E}(Y)$, are

$$
\mathrm{E}(X)=N p s
$$

and

$$
\mathrm{E}\left(Y^{\prime}\right)=N q t
$$

respectively, where $p$ and $q$ are the population rates of clumps of the reference and the test cells in the mixture, respectively, and $p+q=1$.

What is to be estimated in the ratio method is

$$
\frac{\mathrm{E}(Y)}{\mathrm{E}(X)}=\frac{q t}{p s}
$$

The present purpose is to get the sample size $N$, that is, the sum of clumps to be counted to estimate the ratio $\frac{\mathrm{E}(Y)}{\mathrm{E}(X)}$ with a given precision, which depends upon the relative error $\delta$ and the risk of error $\alpha$. In the figure just below, $\frac{\mathrm{E}(Y)}{\mathrm{E}(X)}$ corresponds 
Diagram

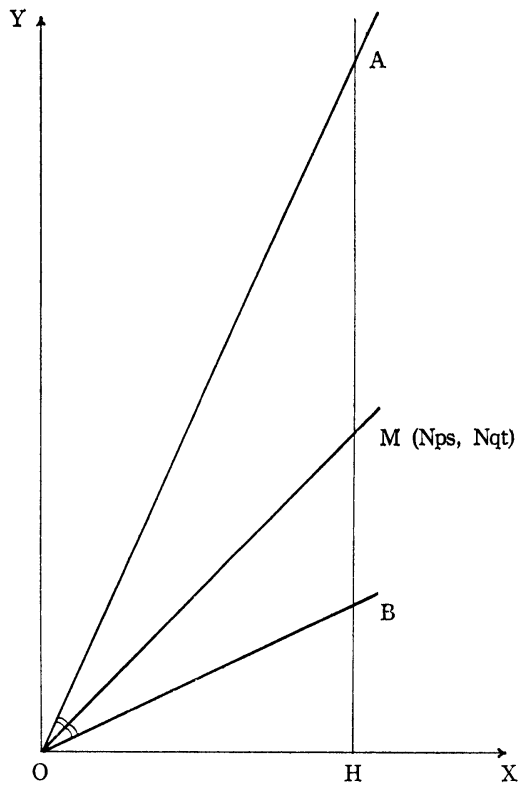

to $\frac{\mathrm{MH}}{\mathrm{OH}}$, and our purpose is reduced to get such $N$ as $\frac{Y}{X}$ will fall between $\frac{\mathrm{BH}}{\mathrm{OH}}$ and $\frac{\mathrm{AH}}{\mathrm{OH}}$ at a probability larger than $1-\alpha$, or

$\operatorname{Pr}\left\{\frac{\mathrm{BH}}{\mathrm{OH}} \leqq \frac{Y}{X} \leqq \frac{\mathrm{AH}}{\mathrm{OH}}\right\}=\operatorname{Pr}\{(X, Y) \varepsilon$ the region bounded by half strait lines OA and $\mathrm{OB}$.

The relative error $\delta=\frac{\frac{\mathrm{AH}}{\mathrm{OH}}-\frac{\mathrm{BH}}{\mathrm{OH}}}{\frac{\mathrm{MH}}{\mathrm{OH}}},(\angle \mathrm{AOM}=\angle \mathrm{BOM})$.

For symplicity, taking the region in which the ratio $\frac{Y}{X}$ will fall to an ellipse which is centered at $\mathrm{M}$ and has the tangents $\mathrm{OA}$ and $\mathrm{OB}$, instead of the above region, an approximate value of $N$ is given by

$$
N \fallingdotseq \frac{4\left(p C_{t}^{2}+q C_{s}^{2}+1\right)}{p q \alpha \delta^{2}}
$$

where $C_{s}=\frac{\sigma_{s}^{2}}{S^{2}}$ and $C_{t}=\frac{\sigma_{t}^{2}}{T^{2}}$

If no clump exists,

$$
N_{0} \fallingdotseq \frac{4}{p q \alpha \delta^{2}}
$$

* Note that from the formula it follows that the sample size is inversely proportionate to $\alpha$ and $\delta^{2}$. 


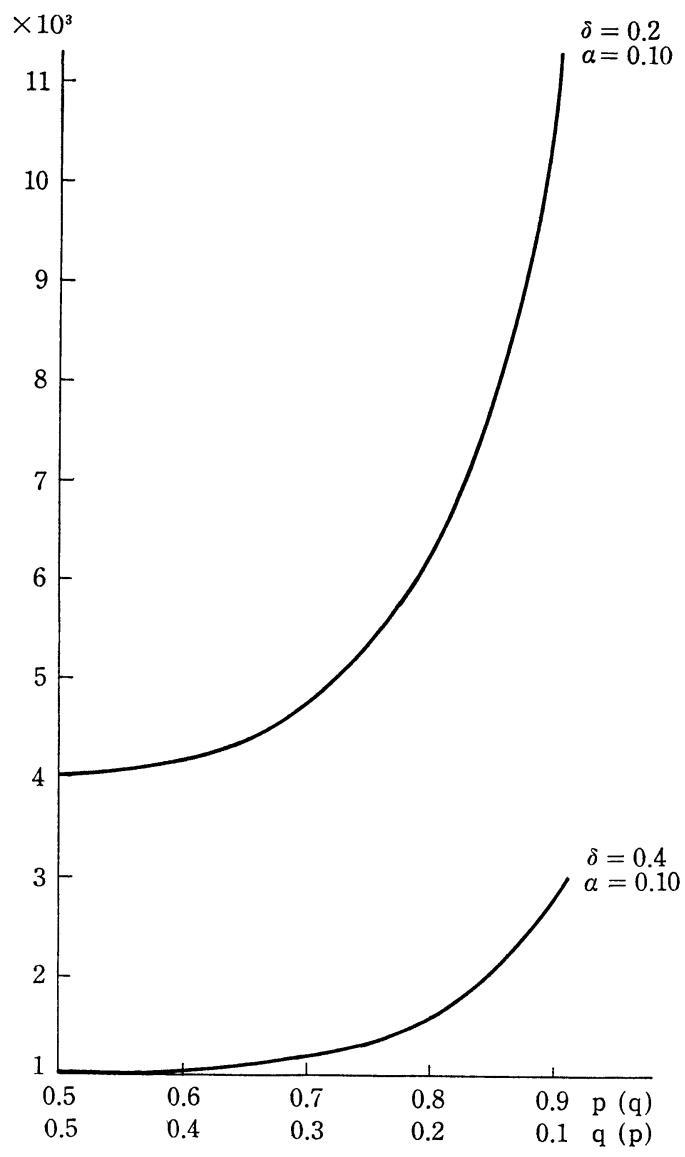

Fig. 5. Examples of relation between the sample size $N_{0}$ and the ratio $p$ to $q$ for two combinations of representative values of $\delta$ and $\alpha$.

$N_{0}\left(\alpha_{1}, 0.2\right)$ for a given value of the risk of error $\alpha_{1}$ at a fixed value of the relative error, say 0.1 , is given by $N_{0}\left(\alpha_{1}, 0.2\right)=\frac{0.1}{\alpha_{1}} N_{0}(0.1,0.2)$. Similarly, $N_{0}\left(0.1, \delta_{1}\right)$ for a given value of the relative error $\delta_{1}$ at a fixed value of the risk of error, say 0.1 , is given by $N_{0}\left(0.1, \delta_{1}\right) \frac{0.2^{2}}{\delta_{1}{ }^{2}} N_{0}(0.1,0.2)$ or $\frac{0.4^{2}}{\delta_{1}{ }^{2}} N_{0}(0.1,0.4)$. See the foot note in page 11.

Examples of the sample size $N_{0}$ are graphically presented in Fig. 5, from which $N_{0}$ for any given values of the relative error or the risk of error will be computed with ease.

So far as the approximate formula just said is concerned, $N$ is minimum when $\frac{p}{q}=\frac{\sqrt{1+C_{s}^{2}}}{\sqrt{1+C_{t}^{2}}} \cdot \frac{p}{q}$ is controlled by selecting a suitable ratio of both the cells.

When $\frac{p}{q}=\frac{\sqrt{1+C_{s}^{2}}}{\sqrt{1+C_{t}^{2}}}$ the formulae (9) and (10) lead to

$$
N=\frac{4}{\alpha \delta^{2}}\left(\sqrt{1+C_{s}^{2}}+\sqrt{1+C^{2}}\right)^{2}
$$


and

$$
N_{0}=\frac{16}{\alpha \delta^{2}}
$$

respectively. Then the ratio of $\mathrm{N}$ to $\mathrm{N}_{0}$ is given by

$$
\frac{N}{N_{0}}=\frac{\left(\sqrt{1+C_{s}^{2}}+\sqrt{1+C_{t}^{2}}\right)^{2}}{4} .
$$

And the sample size $N$ can be computed with the formulae (12) and (13).

Let us consider the following cases to investigate the general aspect of the relation between the ratio $\frac{N}{N_{0}}$ and the situation of clumps.

i) If $C_{s}=C_{t}=C$, that is, in case where both the reference and the test suspensions contain clumps and the variation coefficients are the same,

$$
\frac{N}{N_{0}}=1+C^{2} \text {. }
$$

ii) If $C_{s}\left[\right.$ or $\left.C_{t}\right]=O, C_{t}\left[\right.$ or $\left.C_{s}\right]=C$, that is, in case where one of the suspensions alone contains clumps,

$$
\frac{N}{N_{0}}=\frac{\left(1+\sqrt{1+C^{2}}\right)^{2}}{4} .
$$

Assuming that the distribution of numbers of cells per clump follows the logarithmic series distribution, the relation between the mean of the distribution $\mu$ and the sample size ratio $\frac{N}{N_{0}}$ is given as curves in Fig. 6.

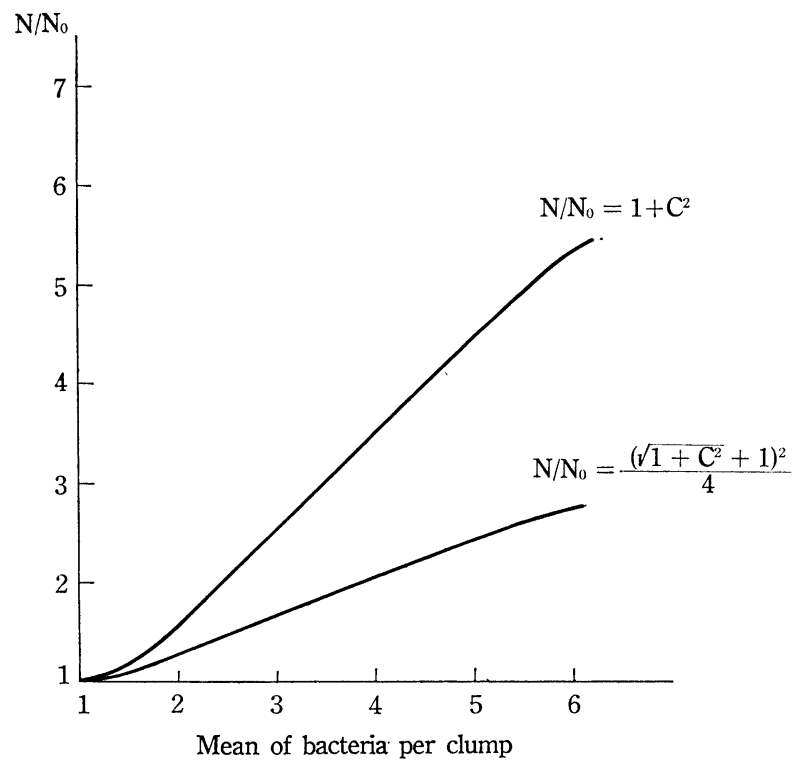

Fig. 6. Relation between the ratio $N / N_{0}$ and the mean of cells per clump.

The upper curve was constructed assuming that both the reference and the test suspensions contained clumps and that the variation coefficients were the same. The lower curve was constructed assuming that one of the two suspensions alone contained clumps. 


\section{DISCUSSION}

A knowledge of the underlying population form, from which the samples were picked out, enables us theoretically to construct tests or estimate parameters for the statistical inference. In the case of data from discrete distributions it may be desirable to verify the form assumed.

Though it has been generally considered that the Poisson distribution was fitted to the frequency of small numbers of bacterial cells per microscopical unit square, this is not always the case, because cell suspensions very often contain some clumps of cells, which give rise a deviation from the Poisson distribution, whether the chamber method or the ratio method is used. Besides, the unavoidably inhomogeneous smearing also makes the mean of numbers of cells per unit square so variable with fields that they deviate from the Poisson. From the theoretically introduced assumptions that the distribution of numbers of cells per clump is a logarithmic series and that the distribution of the mean of numbers of cells per unit square is of the Gamma distribution, it follows that the distributions of numbers of cells per unit square would follow the negative binomial distribution. Our data suggested that this was the case, because the fit often became much better by the negative binomial distribution than by the Poisson distribution.

Similarly, the fit by the bivariate negative binomial distribution to data from the ratio method, in which it is presumed that there is a heterogenous smearing, was demonstrated fairly well. The goodness of fit, however, was not always so satisfactory. It might be due either to incomplete randomization of sampling of observed fields or to some experimental difficulties in the identification of clumps. These problems rising in actual practices, especially in interpretation of clump, are to be taken up in the future.

Anyhow, it can be said that the negative binomial distribution would have a wide application to the distribution of small numbers of bacterial cells per unit square.

That the distribution of numbers of cells per unit square might be considered to follow the bivariate negative binomial suggests that the ratio of numbers of clumps of the reference and the test cells per unit square have remained fairly constant in a random observation. Therefore, the sample size shall be obtained by estimating the probabilities required from the distribution. Since the approach in this way is much complicated, an approximate formula was led by an idea of the Tchebycheff's theorem type, using only the moments of the first and second order of the distribution. The estimates thus obtained may be somewhat larger than that obtained by applying all informations available about the nature of the distribution, though the difference between the two does not seem to be very large. It is noteworthy that the effect of the variation coefficient of distribution of numbers of cells per clump on the sample size is larger than the ratio of numbers of clumps.

\section{SUMMARY}

On the bases of theoretical considerations and experimental evidences, it was shown that the negative binomial distribution would have a much wider range of application to the distribution of small numbers of bacterial cells per microscopical unit square than the Poisson, which has generally been accepted.

And, a modified ratio method was proposed, in which a previously stained and standardized cell suspension from the same species as the cells to be counted was used as a reference instead of the human erythrocytes suspension proposed in the Wright's 
method or an indigo particle suspension in the Thornton's method.

An approximate formula was presented to estimate the sample size to be counted in the ratio method.

The authors wish to express their thanks to Dr. C. Hayashi, Institute of Statistical Mathematics, for helpful advices and for reading the manuscript. Thanks are also due to Dr. S. Iwasa, Mr. S. Asakawa, Mrs. C. Osugi and Mrs. R. Homma, who co-operated in the laborious counting of cells.

\section{REFERENCES}

Arbous, A. G. \& Sichell, H. S. (1954): New techniques for the analysis of absenteeism data. Biometrika, 41, 77-90.

Bliss, C. I. \& FisheR, R. A. (1953) : Fitting the negative binomial distribution to biological data. Biometrics, 9, 176-200.

GuRLAND, J. (1959): Some applications of the negative binomial and other contagious distributions. Amer. J. Pub. Health, 49, 1388-1399.

Jones, P. C. T., Mollison, J. E. \& Quenouille, M. H. (1948): A technique for the quantitative estimation of soil micro-organisms. J. Gen. Microbiol., 2, 54-69.

Joó, I. (1960): Significance of bacterial count in the preparation and potency test of vaccines. Proc. 6th Intern. Congr. Microbiol. Standard., 245-265, H. Horman Verlag, Berlin.

OlitzKI, A. L. (1960): The determination of the number of microorgamisms in a bacterial suspension. Proc. 6th Intern. Congr. Microbiol. Standard., 266-286, H. Horman Verlag, Berlin.

Quenouille, M. H. (1949): A relation between the logarithmic, Poisson and negative binomial series. Biometrics, 5, 162-164. 